\title{
El observador de segundo orden como obstaculizador de la intervención social en jóvenes infractores de ley
}

The second-tier observer as an obstacle to social intervention in young offenders

Carlos GonzÁlez Subiabre

aLicenciado en Servicio Social, Magister en Trabajo Social Universidad Católica de Valparaíso, Chile

$\triangle$ cgonzalez@gmail.com

\section{RESUMEN}

La presente investigación propone una aproximación desde el constructivismo al fenómeno del observador de segundo orden como obstaculizador de la intervención social, particularmente en el caso de los profesionales que trabajan con jóvenes infractores de ley en Chile. Para ello, el estudio transita por diferentes conceptos de orden epistemológico que permiten analizar los problemas en la relación entre profesional y adolescente, además de reconocer los componentes socioculturales presentes en la construcción personal del profesional, con el fin de generar una reflexión sobre las dificultades del proceso de intervención en jóvenes que cumplen condena bajo la ley de responsabilidad penal adolescente.

PALABRAS CLAVE: observador, constructivismo, adolescentes infractores de ley, relación profesional.

\begin{abstract}
This research proposes an approach from constructivism to the phenomenon of secondtier observer as an obstacle to social intervention, particularly in the case of professionals working with young offenders in Chile. To this end, the study uses different epistemological concepts to analyze problems in the relationship between professional and adolescent, besides recognizing the socio-cultural components present in the personal construction of the professional, in order to generate a reflection on the difficulties of the intervention process in young people serving sentences under the law of adolescent criminal responsibility.
\end{abstract}


KEY WORDS: observer, constructivism, adolescent lawbreakers, professional relationship.

\section{INTRODUCCIÓN}

Reflexionar sobre los obstáculos que crea el observador de segundo orden que conoce e interviene socialmente en los adolescentes infractores de ley, en consecuencia se trata de un análisis que se desarrolla en un contexto específico de la intervención social, aquella donde se define como sujeto de atención a estos adolescentes, en un esfuerzo por contener y superar las expresiones del fenómeno de la delincuencia juvenil.

En ciencias sociales, las definiciones epistemológicas permiten generar un marco referencial respecto a cómo entendemos nuestro entorno y cómo desarrollamos un ejercicio, en este caso, de la profesión del trabajo social en el esfuerzo de intervenir la realidad. Esta definición epistemológica -no siempre explicita- se constituye entre un transitar profesional y la asimilación del pensamiento hegemónico positivista expresado en la ciencia y en las instituciones sociales.

Una de las tensiones a la que se expone el profesional es a interactuar entre el pensamiento propio y la visión que domina el contexto institucional (de la institución donde se desempeña), proceso en el cual éste asimila o explora posiciones en su calidad de observador, lo cual condiciona finalmente el contacto directo que se tiene con el adolescente al cual se interviene socialmente.

La existencia de alternativas en la formación del observador, depende de las experiencias significativas y por cierto de la exploración del profesional en su desarrollo académico y reflexivo, siendo absolutamente válida la asimilación y especialización de éste en su posición positivista, pero claramente las intenciones que persigue este trabajo reflexivo es evidenciar parte de lo que se reconoce como tensión a la hora de ejercer e interactuar con la realidad y de forma focalizada con el fenómeno de la delincuencia. El camino que transita esta reflexión busca instalar la complejidad de Morin (2011) como contexto crítico respecto de la forma en que se desarrolla el pensamiento, hipotetizando cómo una epistemología positivista se conecta con el sentido común, manteniendo un discurso hegemónico.

Posterior a ello, la discusión dimensiona los alcances del pensamiento reduccionista y del modelo lineal respecto de cómo la sociedad y sus estructuras observan el fenómeno de la delincuencia, utilizando los aportes de Foucault (2002) con respecto a los medios disciplinarios, lo cual se complementará conceptualmente con la definición de desviación social, otorgando elementos socioculturales en los cuales se desenvuelve el pensamiento reduccionista. Finalmente, a partir de la elección de constructivismo, se intentará explorar y tensionar la expresión elemental de nuestra ejecución profesional, reducida a la interacción con el adolescente infractor, es decir, la relación profesional-adolescente y el contexto que establece, condiciona, la forma y los contenidos de esa relación. 


\section{DESARROLLO}

\section{Naturaleza y alcance del problema}

La posición del observador se constituye en el foco central de este estudio, siendo además, un elemento esencial en el proceso de intervención social que guía los diferentes caminos que pudiese tomar la comprensión del fenómeno y del sujeto de atención. Por su parte, la definición profesional proporcionará los límites y la profundidad de la relación profesional-adolescente.

El ejercicio de definir de forma particular la epistemología, pareciera significar un proceso libre, al menos en su intención. Sin embargo, en el proceso lineal de pensamiento, el positivismo ha abarcado una corriente hegemónica que envuelve la estructura institucional, es decir, la búsqueda del indicador o del verificador como prueba de la realidad.

Esto implica una tensión en la forma en que la institución, a través del Estado, entiende y aborda la delincuencia, en el marco de una política pública y visión economicista. De esta forma, El Estado propone entender el fenómeno de la infracción de ley a nivel adolescente, contemplando modelos reflexivos eco sistémicos y a su vez modelos explicativos objetivables, aludiendo a la epistemología positivista en concordancia con el diseño de las políticas públicas y de sus indicadores de logro.

El profesional, en su condición de observador frente al fenómeno de la delincuencia, trabaja desde un prisma que identifica los desafíos que implica guiar los procesos de cambios en los adolescentes, en primera instancia hacia el desistimiento de la conducta infractora, incorporando en esta visión, no solo las demandas del o los adolescentes en sus distintos tiempos, sino que también las demandas personales de quien se constituye como observador e interventor de una realidad subjetiva.

La Institución gubernamental reconoce en su función normativa la necesidad de diferenciar a quienes se desvían en el contexto de las reglas sociales y cometen acciones tipificadas como delito, atentando contra el equilibrio social, el valor de la propiedad privada y de una sociedad que intenta reprimir acciones de violencia, en todas sus formas, hacia terceros. En este contexto y bajo las reglas del código procesal penal de Chile, en el año 2007 se comienza a aplicar el Sistema de Responsabilidad de los Adolescentes por Infracciones a la Ley Penal (2005), como medio para ejercer socialmente la función de control normativo en menores de edad, estableciendo un sistema disciplinario -concepto que es abordado por Foucault (2002)- diferenciado del sistema penal de adultos de acuerdo a los convenios internacionales que suscribe Chile ${ }^{1}$.

De este modo, la Ley 20.084 (2005) en su descripción inicial definirá la responsabilidad

1 Decreto 830, de fecha 27 de septiembre de 1990, Ministerio de Relaciones Exteriores promulga Convención sobre los Derechos del Niño; Reglas de Beijing, Reglas mínimas de las Naciones Unidas para la administración de la justicia de menores. 
penal de los delitos que cometan los adolescentes, estableciendo para ello la edad de 14 a 18 años y además penas o sanciones especiales en el contexto de esta ley. Es decir, reconoce en los adolescentes su capacidad de discernimiento en la comisión de actos ilícitos, los que deben ser clasificados a nivel penal y con un castigo asociado. Por otro lado, la ley considera dentro de sus contenidos la necesidad de incorporar en todo acto jurídico o administrativo el bien superior del adolescente, consagrado en la Convención Internacional de los Derechos del Niño², por lo tanto, no solo se debe juzgar al menor de edad determinando una eventual consecuencia punitiva por cometer un delito o una acción desviada, sino que además cada decisión que se esboza técnica o jurídicamente debe propender un fin superior otorgando la condición al adolescente como sujeto de derecho.

Las expectativas de la sociedad, expresadas en el marco jurídico y gubernamental, demandan una ampliación del sujeto de atención y la superación de las barreras económicas y sociales asociadas a la comisión de delito. En el artículo 20 de la Ley 20.084 (2005) se establece lo siguiente:

"Las sanciones y consecuencias que esta ley establece tienen por objeto hacer efectiva la responsabilidad de los adolescentes por los hechos delictivos que cometan, de tal manera que la sanción forme parte de una intervención socioeducativa amplia y orientada a la plena integración social" (Ley 20.084, 2005).

En el artículo 20 quedan esbozados los estándares de la institucionalidad respecto de los resultados esperados en una situación de condena, desarrollados por un equipo que interviene al adolescente en un contexto que facilita el proceso de cambio y la integración social.

No es casualidad que la visión positivista mantenga una hegemonía en diferentes ámbitos de acción, ya que de igual forma, se conecta con los sentidos y nuestra interacción con el medio. La mirada se centra en la búsqueda de la evidencia y del objeto tangible, haciendo nuestra comprensión de la realidad como algo medible.

En este contexto explicativo, resulta pertinente considerar el aporte epistemológico de Morin (2011), quien aborda lo complejo y lo reduccionista como parte del proceso interaccional, destacando algunas nociones como tejido, entramado constituido por experiencias, construcciones de símbolos, formas de relacionarse, vincularse e interactuar con diversas instituciones o actores: “...la complejidad es, efectivamente el tejido de eventos, acciones, interacciones, retroacciones, determinaciones, azares, que constituyen nuestro mundo fenoménico" (Morin, 2011, p. 32).

La complejidad que propone este autor remarca que el objeto observado no es independiente de quien observa, a diferencia de lo que plantea la institucionalidad, en la búsqueda de evidencia tangible respecto de los procesos de los jóvenes en el transcurso de sus sanciones. “...La ciencia occidental se fundó sobre la eliminación positivista del sujeto

2 Decreto 830, de fecha 27 de septiembre de 1990, promulga Convención sobre los Derechos del Niño. 
a partir de la idea de que los objetos, al existir independientemente del sujeto, podrían ser observados y explicados en tanto tales" (Morin, 2011, p. 65).

Respecto de su forma de entender el pensamiento humano, Morin (2011) menciona algunos rasgos importantes. Así como la complejidad es “...el desafío, no la respuesta...” (Op. Cit., p. 143), resulta necesario establecer esta propuesta reflexiva, reconociendo en quienes observan, la existencia de fenómenos y el paradigma de la simplificación.

Asumir la complejidad de los procesos sociales es la invitación a no simplificar, pese a que muchas veces se denomina que un fenómeno es complejo en la medida que no sabemos explicarlo. Resulta interesante observar la forma en que frente a un fenómeno complejo como la delincuencia, se toman medidas reduccionistas, por ejemplo la cárcel, o sanciones alternativas. Es decir, las personas e instituciones reducen los fenómenos complejos a datos o antecedentes objetivos, los cuales se transforman rápidamente en las evidencias de discursos hegemónicos y realidades socioculturales. Morin (2004) hace alusión a mandamientos que representan el paradigma de la simplificación, de los cuales se extraen algunos que son pertinentes para este análisis:

“El principio de la ciencia clásica es: legislar; la reducción o también de la elementalidad; la antigua visión, la visión simplificante, es una visión en la que evidentemente la causalidad es simple; es exterior a los objetos; les es superior; es lineal; el pensamiento simplificante fue fundado sobre la disyunción entre el objeto y el medio ambiente" (Morin, 2004).

La aplicación de la política pública, lejos de abordar la complejidad del fenómeno, estandariza los procesos de evaluación y diagnóstico, generando además instrumentos y marcos teóricos necesarios para el tratamiento del desistimiento y la reincidencia del delito. Ello conlleva a familiarizarse con verificadores que fomentan un modelo reduccionista, respecto de la explicación del fenómeno. Un ejemplo de esto es la hipótesis que identifica la deserción educativa como factor que favorece la comisión delictiva y/o reincidencia. El profesional debe trabajar con el adolescente en su motivación e incorporación a un sistema formal de educación, acompañando el proceso de aprendizaje con contenidos académicos y la construcción de nuevas experiencias significativas, así como aprendizajes sobre la vida y oportunidades de crecimiento en el sistema, propiciando que el joven cumpla con las exigencias académicas, supere la condición de deserción del sistema y complete los niveles escolares pendientes en su formación, creando así nuevas necesidades. Si bien, el ejemplo implica un proceso de enormes complejidades, la forma de medir este fenómeno desde la institucionalidad corresponde a la inscripción en algún sistema educacional formal destacando los resultados finales (aprobación o reprobación), siendo éstos datos suficientes para pronunciarse sobre la inserción educativa y los sistemas que tienen mayor éxito (desde lo académico). Esta lógica de pensamiento es transversal a otros ámbitos de la intervención, como el laboral, familiar y de salud. 
Por ello se busca reflexionar en torno a la condición de observador del profesional y los obstáculos que éste mismo genera en la intervención de jóvenes infractores de ley. Esto se tensionará con la visión reduccionista que predomina a nivel institucional, sumado a las representaciones sociales de la delincuencia. Se intentará poner a prueba el modelo sociocultural que contempla la desviación social, como medio explicativo que sustenta la política social de inclusión y las formas de visualizar al adolescente infractor.

Los objetivos del presente artículo fueron: a) reconocer las tensiones del profesional en su condición de observador; b) distinguir subjetividades en la relación profesionaladolescente, desde una mirada constructivista; c) desarrollar un proceso analítico respecto del contexto relacional representado entre el profesional y el adolescente infractor de ley, destacando las limitantes observadas; y d) reflexionar desde el constructivismo propuestas de intervención que permitan visualizar la posición del observador y los límites de la intervención. Con un método cualitativo, el análisis se centró en situaciones de la intervención social en adolescentes infractores de ley en Chile, bajo una mirada teórica basada en autores que permitieron distinguir rasgos susceptibles de estudiar desde la epistemología.

\section{Intervención socioeducativa y castigo}

En la estructura social de Chile se observa -a nivel epistemológico- una hegemoníe del modelo positivista que domina las políticas públicas así como las formas de comprender los fenómenos sociales e intervenir la realidad. El modelo reflexivo de Morin (2011), permite aproximarnos a un contexto complejo, en el cual esboza una explicación, respecto de nuestra tendencia hacia el reduccionismo. Esta tendencia está presente en la construcción de la sociedad, en sus instituciones socio culturales, en la vida cotidiana, en la creación de comunidad y en la conformación del marco legal, vale decir, en el orden social.

Un ejemplo de esto es cómo la sociedad chilena responde habitualmente al fenómeno de la delincuencia, generando temas de discusión cotidiana, mediática y transversal. Independiente de las circunstancias o de los relatos a los que se tiene acceso, las conclusiones tienden a ser similares, con discursos que reconocen el aumento de la delincuencia y que demandan al estado mayor control policial y al poder judicial mayores penas de cárcel. Al respecto, Foucault (2002) sostiene que: "El castigo disciplinario tiene por función reducir las desviaciones. Debe, por lo tanto, ser esencialmente correctivo" (Foucault, 2002, p. 166)

Para crear el contexto correctivo se deben generar espacios de jerarquía, los cuales se encuentran avalados por la estructura y por el ejercicio de la sanción. Ahora bien, en el sistema penal adolescente de Chile la figura del vigilante ${ }^{3}$ no existe salvo en el Régimen Cerrado, pero sólo de forma perimetral. Por tanto, quienes ejercen dicha función en el

Gendarme: Funcionario castrense de Gendarmería de Chile (GENCHI), institución penitenciaria encargada del orden, seguridad, reinserción social, cumplimiento de condenas en las prisiones y el resguardo de los Tribunales de Justicia. Depende del Ministerio de Justicia de Chile. 
medio libre y en el sistema privativo de libertad son los profesionales ${ }^{4}$ a cargo, aplicando la vigilancia desde un enfoque social y educativo.

Al respecto, Foucault (2002) distingue el concepto de examen, el cual opera como mecanismo relacional en el ejercicio de la jerarquía dentro del contexto penal:

"El examen combina las técnicas de la jerarquía que vigile y las de la sanción que normaliza. Es una mirada normalizadora, una vigilancia que permite calificar, clasificar y castigar. Establece sobre los individuos una visibilidad a través de la cual se los diferencia y se los sanciona" (Foucault, 2002, p. 171).

De acuerdo a este autor, el examen permite la utilización de la escritura y la documentación de los procesos observados, abriendo dos posibilidades: a) la constitución del individuo como objeto descriptible, analizable; y b) la constitución de un sistema comparativo que permite la medida de fenómenos globales, la descripción de grupos, la caracterización de hechos colectivos y la estimación de las desviaciones de los individuos unos respecto de otros. En el contexto penal, dada la necesidad de control, la documentación asume la corporalidad y el proceso de cada individuo, en permanente supervisión. De esta forma, cada sujeto se constituye en un caso: “...es el individuo tal como se le puede describir, juzgar, medir, comparar a otros y esto en su individualidad misma; y es también el individuo cuya conducta hay que encauzar o corregir..." (Foucault, 2002, p. 177).

En el desarrollo de los procesos relacionales del ámbito penal los antecedentes que se observan y que consolidan la descripción documentada de cada joven o caso, se utilizan de manera funcional en términos de control. Las reflexiones de Foucault (2002), apuntan a reconocer que el individuo puede ser descrito como el átomo ficticio de una representación de la sociedad. Sin embargo, en esta representación también se incorporan la fuerza movilizadora, significativa y de poder, en relación a lo que se entiende por disciplina, un proceso que se desarrolla en todo tipo de instituciones sociales, especialmente en aquellas que abordan la desviación social.

Sobre el acto de enjuiciar, el mismo autor reconoce que éste es utilizado tanto por los jueces durante el proceso judicial, como por quienes intervienen en el proceso como por quienes ejecutan las sanciones. Si bien, hace referencia a diversos actores que efectúan juicios paralelos, resulta relevante destacar que quienes ejercen la observación presentan de modo permanente la posibilidad de enjuiciar al adolescente o joven infractor. Este hecho no presenta novedad en lo que ya se ha señalado, pero lo complejo es que se da en un contexto donde el sujeto (objeto de atención u observación) ya presenta un enjuiciamiento de la estructura social, con los estándares propios de la desviación social y de la marginalidad.

La observación del profesional estará en mayor o menor medida vinculada con la sentencia original, es decir, con la condición de infractor o desviado social, entorpeciendo

Funcionarios civiles de la administración pública con título profesional. 
así el proceso reflexivo, dado que la concepción del sujeto (adolescente infractor de ley) se encuentra categorizado en el aparato judicial y posteriormente en el contexto penal.

¿De qué forma este contexto condiciona la posición del observador? La estructura social e institucional define a los sujetos de intervención social, a partir de un modelo que categoriza a la sociedad, entre quienes forman parte de la cotidianidad y los que se desvían de las reglas sociales.

\section{Desviación social: Discurso hegemónico frente a la delincuencia}

Las formas de pensamiento complejo sugeridas anteriormente por Morin (2011) reconocen la tendencia individual y social de reducir o simplificar la realidad. Sumado a esto, algunos elementos de Foucault (2002) otorgan estructura al proceso disciplinario de la sociedad y la definición de estándares y mediciones desde lo social. Esta forma de pensamiento forma parte de la definición de las reglas sociales, distinguiendo a quienes se desvían del ordenamiento social. Si bien las reglas sociales buscan ser objetivables, éstas definen conductas y además cambian conforme las sociedades transitan en sus consensos, teniendo de este modo una base subjetiva respecto a la desviación social.

En la ejecución de las sanciones judiciales, el proceso de intervención se encuentra condicionado en parte por las presiones de la estructura social en su conjunto, además de la institución jurídica que demanda esta intervención y que somete al adolescente en el contexto penal exigiendo así aspiraciones más profundas.

Por ello, lo fundamental como observadores es saber distinguir lo que se observa del adolescente en dicho contexto. En relación al observador (profesional civil), este análisis busca generar nuevas preguntas -reconociendo la existencia del prejuicio y del sesgo profesionalcon el fin de establecer nuevas miradas sobre la subjetividad de quien interviene en un contexto de institución pública con procesos estandarizados.

Sobre la delincuencia, se puede sostener que la familia establece los primeros medios de control para que el infante reconozca las reglas o normas sociales, tales como la existencia de la propiedad privada y el respeto a otras personas ¿Se puede entender la desviación bajo un modelo explicativo intuitivo? Sí, cuando se hace referencia a la ciencia moderna y al mandamiento social que construye leyes, según lo que sostiene Morín (2011). A nivel social, las leyes definen la vida moderna, y si bien éstas pueden cambiar, la sociedad se irá adaptando al contexto contemporáneo. Torrente (2001) le otorga un valor importante a este comportamiento social señalando que en las sociedades actuales, reglas formalizadas como las leyes poseen un papel clave para ordenar la vida social. En el mismo ámbito, Becker (2012) profundiza sobre la conducta desviada:

“Todo los grupos sociales establecen reglas y, en determinado momento y bajo ciertas circunstancias, también intentan aplicarlas. Esas reglas sociales definen las 
situaciones y comportamientos considerados apropiados, diferenciando las acciones “correctas” de las “equivocadas” y prohibidas” (Becker, 2012, p. 21).

Ambos autores concuerdan en la aplicabilidad de la regla como estructura normativa, y por ello el factor que resulta interesante para esta investigación es cómo la regla va condicionando la forma de pensar. La paradoja de la simplificación permite reflexionar sobre lo adecuado que resulta la estructuración, la asimilación de leyes (sociales) para distinguir entre lo correcto e incorrecto, y a esto último darle un contexto de desviación. Al respecto, Becker (2012) explica que cuando la regla debe ser aplicada, el supuesto infractor puede ser visto como un tipo de persona especial: “...como alguien incapaz de vivir según las normas acordadas por el grupo y que no merece confianza. Es considerado un outsider, un marginal” (Becker, 2012, p. 21).

El concepto de outsider empleado por Becker (2012) trae consigo un peso importante a la hora de estructurar la forma de pensar el problema y de aislar el fenómeno a un ámbito marginal de pobreza. Bajo esa reflexión, las personas tienden a entender que los motivos de la conducta desviada son un proceso marginal, como si la sociedad no fuera parte de éste. El mismo autor sostiene que "Lo que el hombre común quiere saber sobre los outsiders es por qué lo hacen, que los lleva a hacer algo prohibido y cómo es posible dar cuenta de esa transgresión” (Becker, 2012, p. 23).

La institucionalidad busca la evidencia y genera una segregación a través de esta misma evidencia. Profundizando la forma de pensamiento y la reducción, Becker (2012) explica que la visión más simplista de la desviación es esencialmente estadística, definiendo como desviado lo que se aparta demasiado del promedio.

Por su parte Torrente (2001) incorpora la respuesta social, que resulta interesante desde el comportamiento colectivo cómo se condiciona el pensamiento individual, rescatando que los medios de representación social establecen visiones sesgadas respecto de quienes cometen delito. La respuesta social es generar los mecanismos técnicos que permitan entender la gravedad de infringir una ley, es decir, el rompimiento del orden social. La respuesta de la estructura social debe ser aunada con los diferentes actores, no solo en el plano de las leyes, ya que la desviación incorpora creencias y expectativas sociales. Lo que plantea Torrente (2001) puede ser más drástico:

"Existen diversas áreas de estudios, desde aspectos culturales, psicológicos, sociales que construyen arquetipos individuales, estableciéndose aplicaciones desde lo macro social hacia lo micro social en lo que respecta de lo esperado, de lo correcto, lo moral, por medio de la estructura normativa de la sociedad, categorizando a quienes han infringido la ley, como sujetos desviados socialmente, intencionado nuestra forma de observarlos y de aproximarnos a su realidad" (Torrente, 2001, p. 28). 
Con esta idea, este autor reflexiona sobre el fenómeno de la influencia de las leyes de orden social a la hora de construir la visión profesional del problema, es decir, que el transito del profesional-observador no es inocuo a la hegemonía social. Ya en la búsqueda constante sobre la explicación del fenómeno, la desviación se ha abordado desde el área de salud, entendiendo que sólo una persona enferma, incapacitada, no conectada a la realidad social pudiese cometer una infracción o un delito. Sobre esto, Becker (2012) expresa que “... un punto de vista menos simplista, pero mucho más generalizado, identifica la desviación con algo esencialmente patológico y que revela la presencia de una "enfermedad". Esta perspectiva descansa, obviamente, en una analogía médica". Aquí es donde el discurso dominante genera su marca, a través de un discurso validado desde la ciencia moderna, la cual logra separar al objeto, estableciendo que puede ser observado en su totalidad.

En base a esto, podríamos sostener que la forma de pensar el mundo, se encuentra enraizada en nuestra memoria, en la tendencia a objetivar el conocimiento. "El sentido común es el de la sabiduría tradicional de la tribu, esa mezcla de lo que todo el mundo sabe" dice Becker (2012, p. 207), explicando que al crecer, los niños aprenden los estereotipos de la vida cotidiana, donde también están incluidas las generalizaciones de las ciencias sociales sobre la naturaleza de los fenómenos sociales. Al momento de expresarlos, traducirlos, interpretarlos y acomodarlos en el lenguaje, los contenidos se encuentran integrados de manera subjetiva a la corporalidad. Sin embargo, ingresan a las experiencias de manera objetivada, es decir, hay variados contenidos, datos y narraciones que construyen realidad en un contexto donde cuestionarlo correspondería a una conducta desviada.

La importancia de la desviación como concepto sociocultural, simbólico y referente del pensamiento, es vital cuando el profesional se constituye y evalúa de qué dispone en su estructura cognoscente, para así entender, reflexionar y acceder al fenómeno en cuestión ¿Es posible conocer con cierta objetividad la desviación social? Sería necesario tomar en consideración el contexto penal, el control y la intervención desde aspectos como la marginalidad de los sujetos, la visión o noción de lo reconstruido y la relación del profesional con el cumplimiento de las leyes sociales. Si bien, parece algo resistente, los procesos diferenciadores que impulsen a los profesionales a nivel reflexivo, permitirían la generación de nuevos contenidos y el reconocimiento de diferentes posiciones que ponen a prueba el pensamiento hegemónico.

\section{El observador y la comprensión de sí mismo}

Para analizar las tensiones que enfrenta el profesional en su calidad de observador, vale reflexionar sobre la intervención social con jóvenes infractores de ley en Chile, donde se observa una brecha entre el marco institucional (Estado y Sociedad) y los fines de la Ley $20.084(2005)^{5}$. Esta brecha se basa en un modelo de comprensión positivista que visualiza la

Ley 20.084 que establece un Sistema de Responsabilidad de los Adolescentes por Infracciones a la Ley Penal (2005). Recuperado en http://www.leychile.cl/Navegar?idNorma=244803 
delincuencia juvenil como aquel fenómeno protagonizado por adolescentes que no cumplen los estándares o no poseen las competencias de orden social. En el proceso de intervención se deben revertir esas ausencias y por tanto el fin es convertir a los jóvenes en ciudadanos productivos, siendo ésta una visión que no contempla el fenómeno de la delincuencia como parte de una complejidad, siguiendo la definición de Morin (2011). Uno de los elementos de tensión se observa en cómo reconocemos las brechas del contexto de intervención desde nuestra posición de observadores, siendo estas brechas parte de la misma sociedad chilena que integra y aplica un modelo reduccionista desde lo reflexivo. Conceptualmente se integran elementos que favorecen la simplificación del fenómeno, aceptando su separación de la dinámica social, es decir, la noción que prevalece es que los jóvenes infractores son desviados socialmente y además forman parte de un grupo marginal y excluido del orden social.

Como hipótesis del presente análisis, se plantea que nuestra calidad de observadores se encuentra capturada por el contexto sociocultural, por las estructuras de pensamiento y que naturalmente al no visualizar dicho contexto, la intervención presentará limitantes en el proceso de conocer la desviación social del adolescente infractor de la ley. Ahora bien, las posibilidades de poder ampliar el universo de lo conocido dependerán de la apertura del profesional, y además de una exploración permanente en el ámbito académico y reflexivo, manteniendo así una visión crítica sobre los procesos de la praxis.

El análisis crítico sobre la epistemología positivista puede sustentarse desde diversas bases teóricas, en este artículo, se apostará por aproximarnos desde el constructivismo, centrando el foco en la posición del observador, reconociendo a su vez la subjetividad presente en el proceso de conocer la realidad. Pensarse como observador, permite reflexionar sobre las cualidades, los límites, la relación con el objeto, y declarar una epistemología. En palabras de Maturana (1995): "Los seres humanos nos descubrimos como observadores de la observación cuando comenzamos a observar nuestra observación” ${ }^{6}$, siendo éste un ejercicio que posibilita a través del lenguaje construir lo que observamos. Reconocernos como observadores implica distinguir los límites existentes respecto del conocer, junto con identificar el prisma epistemológico que genera los significados.

Desde el construccionismo podemos señalar que desde el primer contacto uno de los elementos que conecta y alimenta la relación entre profesional y adolescente es el uso del lenguaje. En este sentido se destaca la forma de conectar a los contenidos y significados del adolescente, siendo uno de los medios la narrativa, tal como lo explica Maturana (1995): "Los seres humanos existimos en el lenguaje, y nuestra experiencia como seres humanos se produce en el lenguaje, en el flujo de las coordinaciones

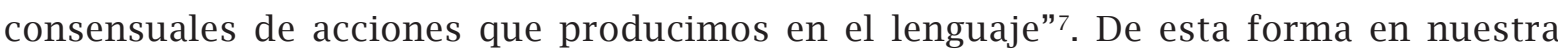
condición de observadores, la conexión se materializa a partir del relato y de las

6 Maturana, H. (1995). La ciencia y la vida cotidiana: La ontología de las explicaciones científicas. En P. Watzlawick \& P. Krieg (Comps.), El ojo del observador. Contribuciones al constructivismo. Barcelona: Gedisa.

Op. cit. p.187 
subjetividades que encierran la relación. Para profundizar las subjetividades desde una perspectiva constructivista se desarrollarán algunos contenidos metafóricos, partiendo por la posición del observador.

La discusión respecto de la existencia de la objetividad, ha permanecido en el escenario postmoderno, en donde en algunos casos se busca otorgar validez a la corriente positivista y constructivista. Aguirre y Jaramillo (2010) se basan en Fodor para sostener que la observación es un tipo de inferencia y acuerdo de que en la percepción hay un aislamiento radical en cómo se ven las cosas de los efectos de todo lo que uno cree.

Otra reflexión drástica respecto de la imposibilidad de conocer la realidad es aportada por los mismos autores citando a Dretske para aseverar que no podemos comparar ni nuestras experiencias o creencias acerca de la realidad con el modo de cómo es la realidad: “...pues en orden a mirar si nuestras experiencias o creencias corresponden con la realidad, siempre otras experiencias o creencias deben intervenir" (Aguirre \& Jaramillo, 2010, p. 79).

El reconocer que la observación depende de la teoría, o del contenedor conceptual de donde proviene, determina su origen subjetivo. Así mismo, la capacidad de ser observador y mantener una posición neutral fuera de prejuicio no es posible, dado que no es posible escindirse de las teorías que se contienen y por ello lo que se escoge finalmente como conocimiento científico desde el observador será una elección subjetiva.

Aguirre y Jaramillo (2010) analizan la carga teórica existente en el proceso de observación, y en un contexto constructivista, rescatando que aunque la observación esté cargada de teoría no implica que sea imposible la objetividad del conocimiento, concluyendo que la observación es falible y que no puede darnos un fundamento cierto y seguro.

En esta reflexión ambos autores establecen un punto más cercano a consensuar respecto del relativismo extremo y la ausencia de toda objetividad y disciplina que lo organice. Citando a Sankey expresan que los “...presupuestos teóricos que guían la observación pueden hacer que evidencia importante sea pasada por alto, así como pueden hacer que a la evidencia irrelevante se le dé un significado inmerecido" (2010, p. 80), recalcando que la contaminación de la actividad perceptual por la teoría significa que un hecho observado puede malinterpretarse debido a la influencia de la teoría sobre la interpretación del hecho. Se reconoce entonces que la existencia de un contexto teórico nos limita en el proceso de conocer, sin descartar que los mecanismos que externalicen la observación permitan una construcción de la realidad más consensuada. Esta perspectiva pondría a prueba los conocimientos, fundamentos teóricos y prejuicios de los profesionales, permitiendo además el desarrollo de una intervención supervisada que revise el tipo de modelos explicativos que se están aplicando. Este enfoque de la intervención se vería favorecido por la estructuración de intervenciones coordinadas en equipo y la realización técnica de análisis de casos entre quienes intervienen con el joven, en situaciones donde no sólo se busquen acuerdos, sino que también se identifiquen las distinciones y las construcciones que elabora el equipo respecto del adolescente. 


\section{Aproximaciones constructivistas hacia la relación profesional-adolescente}

En esta reflexión se ha generado un diálogo entre varios conceptos sobre la construcción de la calidad-cualidad del observador y en especial, las condiciones de su observación, donde se ha distinguido que el observador no está separado de su observación, ni de lo que observa. Otra condición se relaciona con el entramado social y simbólico, más el tejido en el que se encuentra el profesional en la observación y relación terapéutica. Dada la auto-referencia racional y constructivista, el sujeto tiende a vivir las cosas como protagonista y centro de todas sus experiencias y de las otras personas con que interactúa.

El observador también posee contenidos relacionados con la cultura y el aparato disciplinario de la sociedad al cual se encuentra sometido, vale decir, lo relacionado con la demarcación de lo correcto y lo incorrecto, junto con el sometimiento hacia la mantención de equilibrio institucional y la asimilación del discurso social dominante, respecto de la desviación y la marginalidad social vinculadas directamente a la comisión de delitos. En la intervención con jóvenes infractores, se visualiza al observador (profesional) desarrollando su lógica de pensamiento y contacto con el sujeto en un contexto institucional penal, donde los juicios profesionales son vinculados al proceso observacional, y se elaboran sobre aquello que es juzgado penalmente. Por otro lado, el observador interviene en un contexto normativo y punitivo con el cual debe tener coherencia en la línea de acción acorde con la institución, ya que podría ir en otra dirección con respecto al proceso de cambio que se espera a nivel social, vale decir, corregir la conducta desviada.

¿Los profesionales que intervienen en estos contextos se encuentran en un espacio privilegiado para entender esta complejidad? ¿Al profesional en su proceso de exploración y aprendizaje, le será más funcional separarse de la reflexión epistemológica y aproximarse a modelo ecléctico que permita acomodar los desafíos de la intervención y de la institución?

Desde el contexto terapéutico, White (1993) analiza la posición del observador guiándose por Foucault, destacando que el uso del poder no necesariamente está vinculado a características de índole represiva o controladora, sino que también a efectos positivos asociados, siendo un factor constitutivo respecto del funcionamiento de nuestras vidas. Sobre esto señala lo siguiente:

“...el poder es constitutivo o determinante de las vidas de las personas. La idea de un poder cuyos efectos son negativos aporta una teoría de la represión, mientras que la idea de un poder cuyos efectos son positivos conduce a una teoría acerca de su papel en la 'construcción' de las vidas de las personas" (White, 1993, p. 36).

La construcción de verdades normaliza la configuración de nuestras vidas, sostiene White (1993), basándose en Foucault para defender la creencia en ideas construidas a las que se asigna un status de verdad. "Estas verdades son 'normalizadoras' en el sentido 
de que construyen normas en torno a las cuales se incita a las personas a moldear o constituir sus vidas" (White, 1993, p. 36). Desde aquí, el autor remarca la importancia de familiarizarse con la visión de poder, especialmente desde su funcionalidad cotidiana, y principalmente desde su importancia para el proceso terapéutico.

Elementos importantes a considerar son las factibilidades que se observan en el proceso terapéutico del cambio y la desnaturalización del positivismo como único medio válido de intervención. Shaefer (2014) señala que en psicoterapia existen caminos hacia la imposibilidad del cambio que van vinculados a las bases epistemológicas para la intervención. Entre esos caminos, reconoce que el uso de etiquetas diagnósticas podría condicionar las expectativas dentro de ese espacio, o bien, la existencia de la contratransferencia de la teoría, haciendo referencia a los amarres o estructuras propuestas por el modelo teórico.

Shaefer (2014) resalta además la relevancia de los factores comunes en el cambio terapéutico, que dependen de la participación y protagonismo del paciente. Esto genera permanente tensión, en el sentido de que la intervención se da en un contexto forzado hacia los adolescentes, es decir, deben asistir a un programa/sanción y someterse a la posibilidad de cambio, sin considerar el contexto actual ni sus principales necesidades.

Si bien, ya reflexionamos en torno al contexto subjetivo de la intervención y del observador, queda por abordar el impacto que recibe el profesional de los cambios o experiencias del sujeto. Szmulewicz (2013) explica que participar de los relatos de vida de los pacientes enriquece la propia vida del terapeuta desde un punto de vista constructivista, ya que permitiría "...incorporar nuevas narrativas sobre sí mismo, sobre el paciente y sobre otros pacientes...” (Szmulewicz, 2013, p. 62). En el caso de profesionales que intervienen con jóvenes infractores desde las sanciones penales, las alternativas que se pueden dar son diversas, tomando en cuenta que a través de la intervención se desarrolla una dicotomía, la que se expresa a partir del rol contemplado en la ley y en la institucionalidad, es decir, por un lado el control de la sanción desde un plano normativo, y por otro, la plena inserción social y el bien superior del adolescente. Las posibles respuestas que vaya identificando el profesional se definirán en la forma de cómo se vaya desarrollando dicha dicotomía. Un factor esencial, dice relación con la posible desensibilización de los procesos desde el mismo profesional, a propósito de las dificultades vinculares propios del contexto y la normalización de los resultados negativos, las recaídas, las pautas violentas que expresan los jóvenes, la persistencia de la conducta delictiva, los intentos fallidos de los jóvenes por lograr metas prosociales. Se podría decir que los profesionales se enfrentan a permanentes obstáculos, propios de la intervención y del contexto sociocultural del joven.

En este espacio, en donde se construye el observador/profesional, sus respuestas no pasan desapercibidas, ni son inocuas frente a los nuevos relatos que va construyendo. Al respecto, Szmulewicz (2013) explica lo siguiente: 
"El terapeuta puede reescribir y cambiar significados simbólicos de ciertos acontecimientos de su vida a partir de la resignificación que hacen los pacientes de su propia vida. La actividad terapéutica repercute en la relación que tienen el terapeuta con el mundo y en sus relatos de identidad personal, así como en la atribución de significados que hace de sí mismo, de su vida y de su trabajo" (Szmulewicz, 2013, p. 62).

En relación al desarrollo del juicio profesional, la tendencia técnica en el contexto institucional es buscar evidencias que respalden las decisiones profesionales, y en este contexto las narrativas de los adolescentes son puestas a prueba constantemente. Sin embargo, la apuesta de intervención sugiere permanentemente generar procesos vinculares, pero no se considera cuando se pone a disposición desde el profesional para la construcción del vínculo. Guiándose por Stoloorow y Atwood, Szmulewicz (2013) sostienen la inexistencia del terapeuta neutral, refiriéndose al terapeuta mismo como aquel que interviene desde sus propios prejuicios y convicciones, interactuando con los del paciente permanentemente. Comprender y asumir la subjetividad desde el profesional, naturalmente es un comienzo para el proceso transformador, especialmente para la apertura profesional.

Esta comprensión facilita entrar en el escenario de la intersubjetividad, lo cual convoca a reconocer el intercambio producido en la relación profesional/adolescente. La conciencia del proceso debe ser liderada por el profesional que interviene, quien además la capitaliza como insumo en la construcción. Desde la perspectiva terapéutica se generan algunas corrientes para trabajar la horizontalidad de la relación para reconocer la subjetividad propia, pero sin renunciar ni desconocer el rol ni el poder configurado en la relación. Szmulewicz (2013) explica que la intersubjetividad siempre “...implica una dialéctica entre (re) conocer al otro y ser (re) conocido por otro" (Szmulewicz, 2013, p. 62). Este mismo autor recalca que ambos propósitos operan también en el terapeuta generando una tensión que lo hace evitar entrar en un camino de autodevaluación.

El reconocimiento de la subjetividad desde el terapeuta en si no es una novedad, pero de acuerdo a Szmulewicz (2013), lo importante es recabar y describir los elementos que constituyen la relación, tomando en cuenta la nueva experiencia. El proceso de conocimiento personal resulta relevante para distinguir la subjetividad propia, frente que lo que Szmulewicz (2013) señala que el camino hacia el conocimiento personal es un proceso sin fin, pues mientras se viva se sigue teniendo experiencias y el ensamblaje terapéutico también es continuo, de modo que “...la tarea de ver/entender al otro va unida con la tarea verse/ entenderse" (2013, p. 63).

Incorporando otra perspectiva, el observar lo que se observa, conlleva un proceso permanente de auto revisión, específicamente durante el intercambio de significados, junto con incorporar la visión de intersubjetividad dentro de la relación profesional/adolescente. Ahora, todo lo anterior resulta complejo de sostener, ya que el profesional debe contemplar rupturas (o crisis) en la relación. Vale decir, es comprender que su posición como terapeuta 
condiciona también las posibilidades de vinculación, por lo cual resulta difícil esconder que las rupturas no tengan directa relación con la figura del terapeuta o con las subjetividades del profesional. Basándose en Winnicott, Szmulewicz (2013) establece que el terapeuta es quien debe cuidar la zona intermedia y el manejo de la confianza. Estas observaciones son relevantes a la hora de definir caminos acertados para lograr un mejor proceso de cambio, toda vez que la relación intersubjetiva se da en contexto normativo, en donde no sólo como profesional se representa así mismo, sino también representa a los jueces, a la víctima, a la condena, a la sociedad, por lo cual las posibilidades de que se produzcan rupturas en el vínculo son reales. La desconfianza de los jóvenes hacia la figura del profesional, es parte permanente de las resistencias hacia la intervención, lo cual se ve entorpecida por el ejercicio del control normativo y por representar a la institución ejecutora en los procesos de control judicial de la sanción.

García (2008) se refiere a lo inevitable de los cambios que genera la interacción especialmente en un proceso terapéutico, resaltando que la mirada del otro de alguna manera nos inoportuna y nos fuerza a reinventar nuestra propia realidad: “...el conflicto es inherente a la relación porque nos construimos en el contacto con los demás y cualquier contacto con ellos nos obligará a reorganizar nuestro modo de ver el mundo” (García, 2008, p. 362).

Los procesos culturales contribuyen a la creación de significados, ya que desde el punto de vista constructivista y según García (2008), resulta importante entender que -en el ejercicio de comprender al otro- los rasgos culturales influyen en la elaboración del significado. De ahí que las conductas infractoras y las formas de relacionarse a través de la violencia como medio, dependerán de los diferentes contextos que experimente el adolescente.

De acuerdo a García (2008), desde el constructivismo la elaboración de significados se entiende como un diálogo entre un marco cultural de conocimiento y un sujeto que construye relacionalmente una interpretación personal de dicho marco cultural. Esto no reviste un ejercicio simple, ni un recetario paso a paso para contactarse con el contenedor cultural de la subjetividad de un adolescente, pero el proceso reflexivo resulta fundamental para construir aproximaciones a los significados sobre una representación cultural respecto de los jóvenes y tener mejor sintonía con sus prioridades, sus intereses, y así no sólo quedarnos con la visión de la desviación social. Para explicar esto, García (2008) sostiene para entender el mundo, es necesario entender al otro, para lo cual hay que ser capaz de adecuar la representación de su conducta y visión del mundo en términos de los propios constructos. Si pensamos al sujeto como cuerpo contenedor de subjetividad y constructor de significados, se tendría que desarrollar una revisión de lo que el cuerpo contiene. Desde el nacimiento, a través de la infancia y hasta alcanzar la adolescencia, tenemos a un sujeto que presenta una expresión desde su corporalidad presente, pero que dice relación con una gran cantidad de acontecimientos, experiencia y significados construidos/reconstruidos según se acumula el proceso de conocer. 


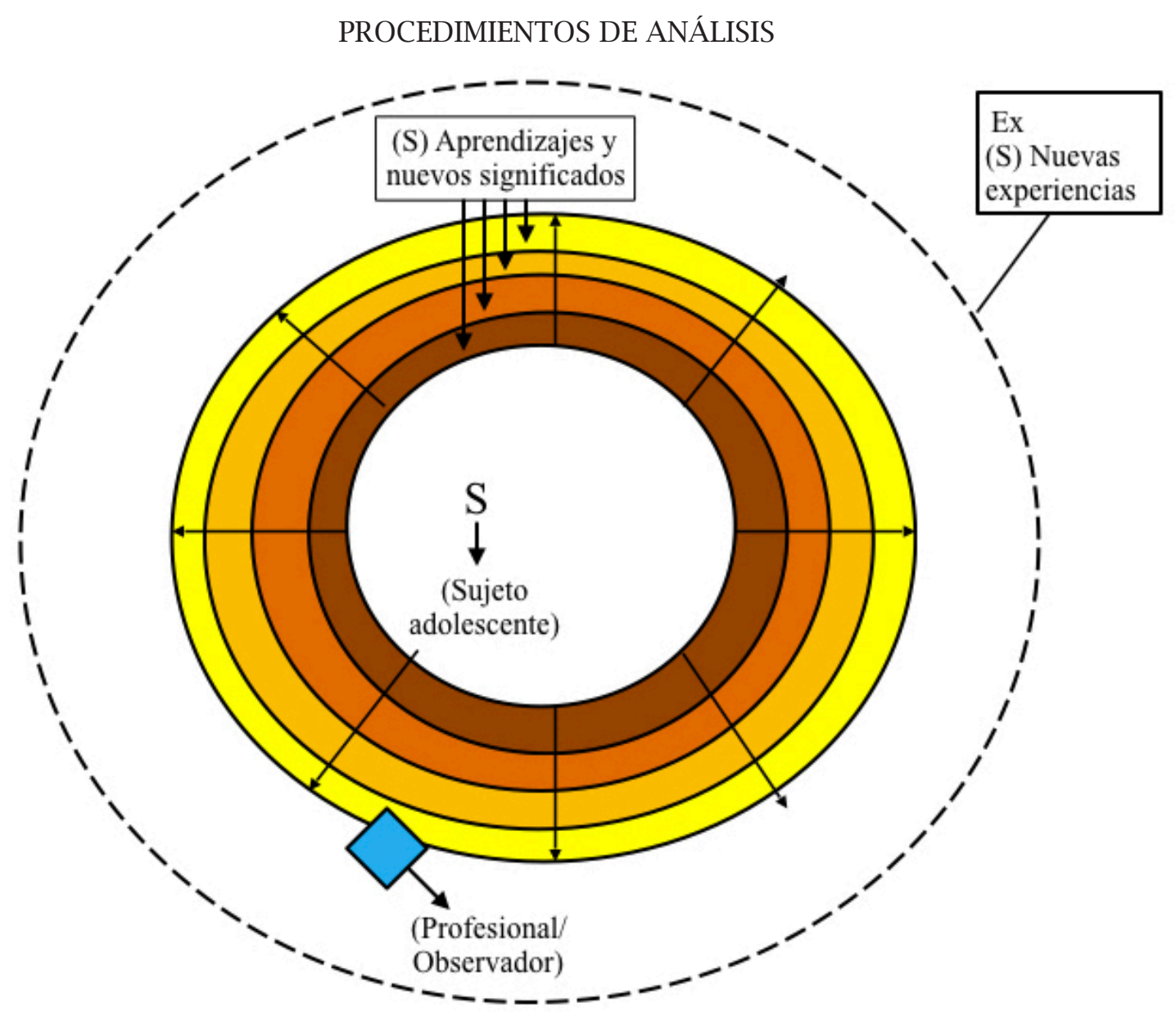

Fig. 1. Construcción desde la experiencia del adolescente en el contexto de encuentro con el profesional. Fuente: Elaboración propia.

En la Fig. 1, la letra S corresponde al sujeto adolescente, quien en el tránsito de su vida va acumulando un conjunto de experiencias en un proceso de historicidad en la construcción de significados, junto a la maduración y adaptación del cuerpo frente a las experiencias de la vida. En resumen, el sujeto va transitando y generando una nueva capa que configura los significados, los cuales se condensan en un nuevo cuerpo de éstos mismos.

Cada evento le da un matiz diferente al cuerpo, es decir, cada cicatriz -física o emocional- transforma, construye/reconstruye la identidad. En cada circunferencia nueva (o capa) se van conteniendo las experiencias, aprendizajes y nuevos significados, adquiriendo así una nueva forma y color.

La presencia del profesional, ya sea como delegado, trabajador social o psicólogo en el contexto de justicia juvenil, se encuentra representada por el rombo de color calipso, en una ubicación con claras potencialidades de influir en la formación del nuevo cuerpo 
o capa del sujeto adolescente, lo cual dependerá del nivel de integración alcanzado en el proceso. Las oportunidades de intervención están situadas en la frontera de las nuevas experiencias (Ex) del esquema, donde el adolescente podría integrar al profesional de forma significativa.

La necesidad de comprender la mirada y significados del adolescente infractor está lejos de aportar a la configuración de una visión desde la victimización del delincuente, reduciendo así su responsabilización respecto de los delitos cometidos, ya que por el contrario, la idea es generar un espacio analítico que permita dimensionar las respuestas y el contexto al cual se enfrentan en la búsqueda del cambio, es decir, ampliar los parámetros explicativos y obtener varias opciones para la intervención. Es importante, de igual modo, reconocer que los adolescentes también se encuentran representados por su corporalidad, ya que el cuerpo expresa parte de lo que se ha vivido, como por ejemplo la agresividad que expresan los jóvenes tanto entre sus pares como hacia las personas que los rodean, sumado a esto, la ausencia de empatía hacia terceros, especialmente a la hora de cometer nuevos delitos, observándose de igual forma una capacidad diferente de relacionarse con el dolor físico, creyendo ellos mismos que poseen un mayor umbral de dolor que las otras personas.

Al respecto, García (2008) reflexiona que la falta de empatía en jóvenes infractores se relaciona con el sistema de significados que poseen, siendo éstos inestables e impredecibles, describiendo sus relaciones con el entorno por medio de tres características: a) Un mundo inestable, que no permite una reflexión a medio o largo plazo, b) un mundo inestable es destruible; y c) dificultades para establecer relaciones de intimidad estrecha con los demás.

Los principales conceptos que se buscan aplicar en las reuniones de coordinación entre profesionales del área de justicia juvenil se enfocan en el vínculo, vale decir, el nivel de vínculo que el joven ha alcanzado a desarrollar en un contexto penal con profesionales asignados para el control, los cuales, intuitivamente buscan encontrar indicios respecto de la capacidad de vincularse que poseen los adolescentes, entendiéndolo como una puerta de entrada al área emocional del joven, es decir, un acceso al círculo de confianza. Lo complejo es la búsqueda y construcción de una relación terapéutica con fundamentada en el vínculo, en un contexto en donde parte de la función es representar a la institución ejecutora de la sanción y ser parte del control.

Las expectativas profesionales y los procesos técnicos pueden ser apresurados en relación a la construcción que puede elaborar un adolescente especialmente en contextos ambivalentes de intervención. Naturalmente, el profesional busca cualquier indicio que permita dimensionar al joven no sólo desde la desviación, sino también desde su emocionalidad y desde el sujeto relacional. Uno de los elementos a revisar respecto del proceso de intervención, es que sucede cuando un joven demuestra lo contrario, es decir, expresa agresividad, mantiene distancia, y no genera acciones que favorezcan el vínculo. 


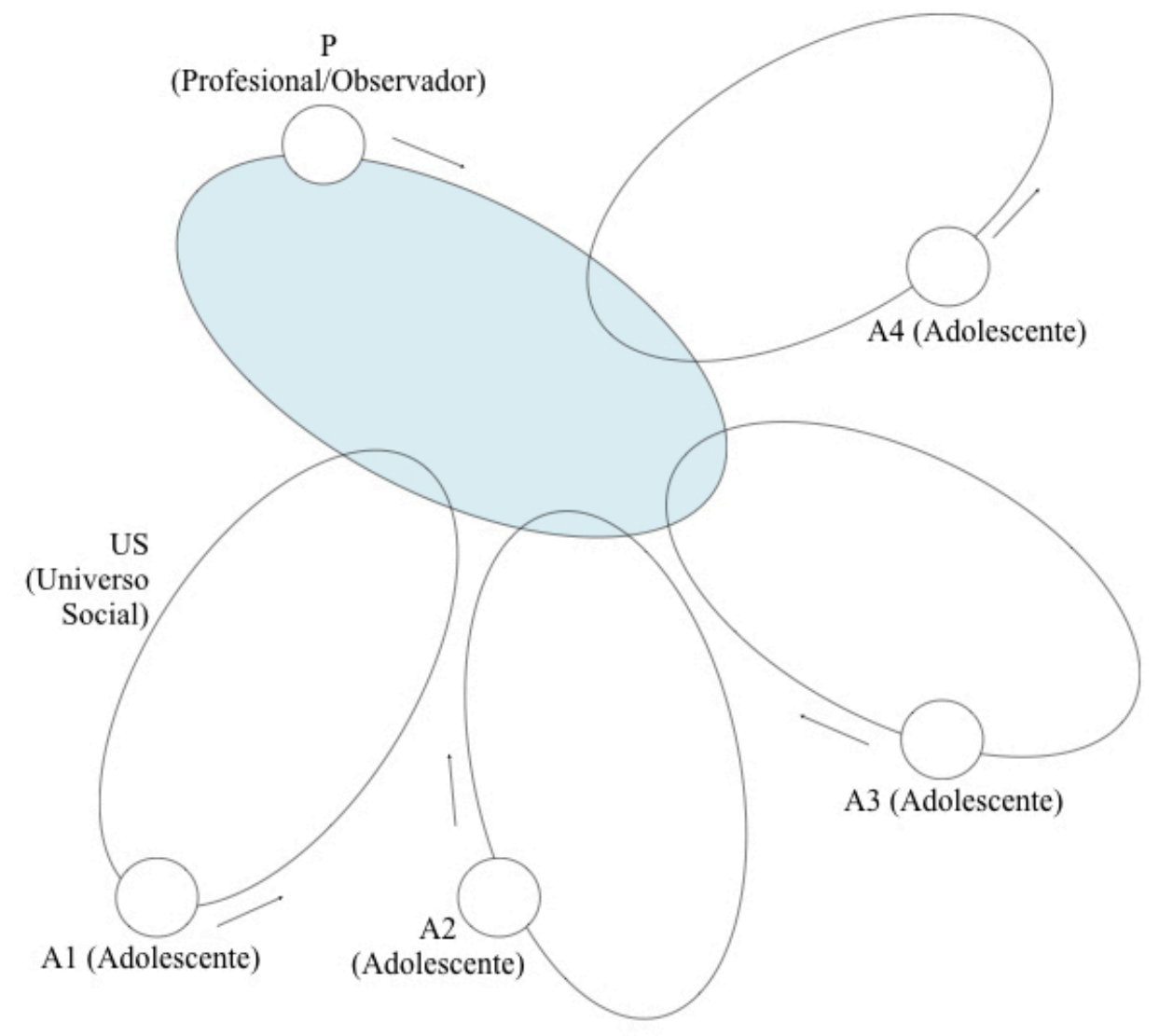

Fig. 2. Orbita del profesional/observador. Fuente: Elaboración propia.

En la Fig. 2 se aplica el concepto del giro copernicano ${ }^{8}$ a la relación que establece el profesional (P) con el adolescente (A1, A2, A3 y A4) en el contexto de sanción, podríamos pensar que quienes ejecutan las sanciones giran en la esfera de lo social, en un pequeño cuadrante del universo social (US) conocido. Si representamos al profesional como un planeta, este sigue una órbita determinada, según su contexto social e individual. Una forma de interpretar la dinámica relacional, es que el profesional dentro de su órbita coincide con la órbita de algunos jóvenes por un período incierto. Bajo el supuesto de que el profesional no es el centro del universo conocido, se podría comprender que los adolescentes en su propia orbita podrían tener definidos sus propias versiones, nociones y discursos respecto de la institucionalidad penal, tanto de quienes sancionan como de quienes ejecutan condenas.

Este ejercicio, intenta representar de qué forma el profesional y el adolescente

8 Metáfora con que se alude al cambio radical de perspectiva que supone, respecto de la filosofía tradicional, el planteamiento general de la filosofía de Kant, y al cual él mismo alude en el Prefacio de la Segunda edición de la Crítica de la razón pura. El «giro» -término que proviene de la imagen de las estrellas que giran alrededor del espectador- que Kant es consciente de llevar a cabo es similar al que hizo Copérnico, quien supone que es el espectador el que gira en lugar de las estrellas. Fuente: Cortés, J. y Martínez, A. (1991) Diccionario de filosofía. Barcelona: Herder. 
podrían interactuar dentro de una relación y sus potenciales posibilidades de intercambio, de crecimiento, rechazo o acoplamiento. De igual forma se busca resaltar la permeabilidad bidireccional entre profesional y adolescente.

Tal como se intenta representar la tendencia respecto de cómo se comprende la intervención, los jóvenes (A1, A2, A3 y A4) asignados a un profesional (P) destinan parte de sus vidas a una sanción penal, en un contexto definido por el universo social (US) conocido. El sentido de la interacción, guiada y contenida en una estructura social, no sólo busca formar parte del cuerpo institucional de justicia y controlar la ejecución de una condena, si no que busca de acuerdo a los fines de la ley y a los componentes éticos de la profesión, generar acciones que concluyan en un impacto favorable de plena integración social, lo cual debe ser generado durante el tiempo que se produce la intersección.

En la Fig. 3 también se aplica el concepto del giro copernicano, junto con las nociones de profesional/observador (P), adolescente (A3) y universo social (US), observándose así que el contexto relacional se configura desde los espacios que posee la interacción. También se marca el origen de tal relación, es decir, desde la entrada en vigencia y hasta el fin de la sanción penal. Si las respuestas del adolescente frente al mandato judicial no resultan inocuas para el profesional, éste podría definir su posición en este espacio, ya sea impactando o acoplándose

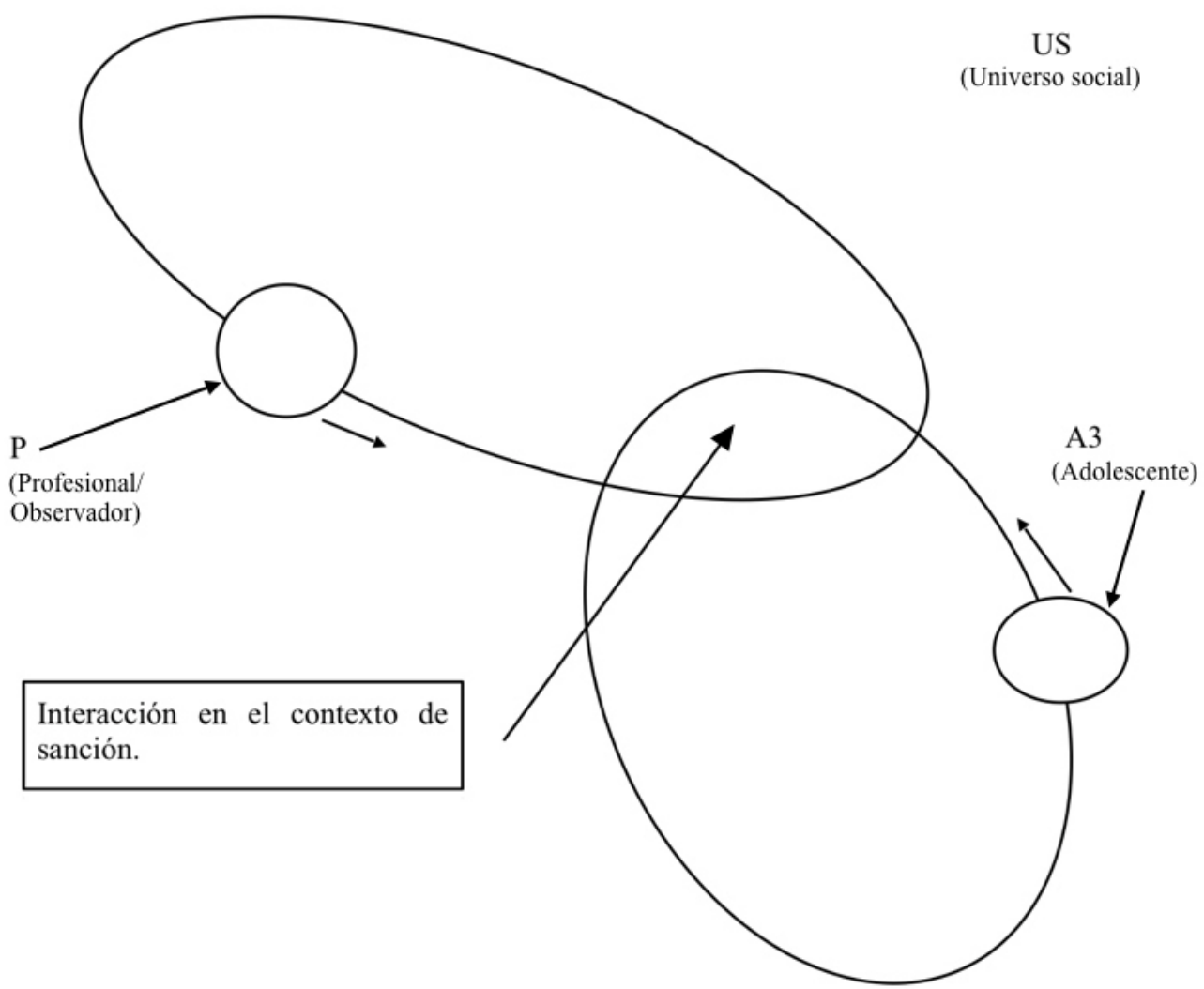

Fig. 3. Orbitas de la relación profesional-adolescente. Fuente: Elaboración propia. 
a la órbita del adolescente, existiendo además la opción de asumir que el adolescente debe ajustarse al mandato judicial y por ende a la órbita institucional.

Este proceso se puede asociar a un eventual resultado para ambos sujetos (profesionaladolescente), para la institución y las estructuras sociales que cobran una forma orgánica, a través de jueces, defensores, fiscales, supervisores institucionales, medios de comunicación, opinión pública, más individuos cercanos o significativos para el joven, todo esto con la finalidad de conocer los logros obtenidos.

Lo complejo del proceso relacional esperado en este contexto corresponde al resto de la órbita del joven, vale decir, quienes y qué lugares componen esta órbita, ya sea en sus momentos de ocio, planificación, vicios, en las reuniones con sus hermanos de la calle, en el delito, con sus parejas, junto con los ámbitos de sus metas, juegos, alegrías y tristezas. Estos elementos en su conjunto alimentan la construcción de su identidad, pero el profesional sólo alcanza a observar lo que el adolescente le permite acceder en dicha intersección de ambas orbitas, es decir, la interacción en el contexto de sanción.

\section{CONCLUSIONES}

La presente reflexión busca incorporar elementos epistemológicos y teóricos de manera aplicada a la experiencia profesional en el contexto de la intervención social a adolescentes infractores de ley en Chile. En base a los antecedentes ya expuestos, se puede sostener que el enfoque constructivista no separa al objeto del sujeto, así como la sociedad no se separa de la marginalidad o la pobreza. El contenedor sociocultural, dentro del contexto marginal, cuenta con representaciones sociales significativas vinculadas a la comisión de delitos, incorporándola como una alternativa identitaria válida, reconocida y funcional a nivel social. Es importante admitir que el observador debe explorar las representaciones de la sociedad por medio de sus instituciones, junto con las representaciones de adolescentes bajo el supuesto que éstas formarán parte del proceso relacional entre profesional y adolescente. Esto interfiere en el proceso vincular, en el acoplamiento de ambos sistemas y en las expectativas de cambio. Por ello, no resulta extraño que las mismas instituciones o la sociedad limiten tal interacción, al expresar pautas o sesgos sobre cómo observar el fenómeno.

\section{Observador como centro de la intervención}

Si bien, son necesarios más estudios para analizar el fenómeno de la complejidad que enfrenta el observador en la intervención social de adolescentes infractores de ley, en este estudio se plantea la necesidad de confrontar las reflexiones de cada caso, es decir, admitir que conocer al sujeto adolescente depende de qué pone a disposición el observador/profesional y no asumir que dada la relación de poder que ofrece el contexto penal, social e institucional, el adolescente se subordinará al proceso. Por ello, situarse 


\section{GonZÁLEZ}

como observador en un determinado universo social, supone la existencia de accesos a ciertas realidades, lo cual podría ser o no consensuado entre diversos observadores del mismo universo social, lo cual da a entender de que existen niveles de profundidad en la vida de cada sujeto que no logramos comprender. Este enfoque libera al observador y le permite navegar en el universo social con las expectativas centradas en explorar y conocer para favorecer la comprensión y aceptación del otro, siendo éste el punto de partida para iniciar el proceso de cambio.

\section{Observador como contenedor sociocultural}

Cuando se configura al observador dentro del plano profesional, la coherencia constructivista no se da en todos los planos, dado que la forma de relacionarnos con el mundo se sustenta en el pensamiento reduccionista. Por ello, es clave transitar desde lo consciente hacia lo objetivable por medio de un análisis crítico, es decir, que desde nuestra subjetividad -como intérpretes de las experiencias- contamos con esa distinción respecto al conocimiento que adquirimos. Deshacerse de ciertos contenidos para volver a integrar otros, es un proceso que nos permite pensar en la incorporación de nuevos conocimientos. Sin embargo, prescindir de los contenidos de toda una vida parece algo poco factible y por tanto se debe asumir que nuestras experiencias se encuentran contenidas en el cuerpo físico, como contenedor que refleja parte de lo vivido. Es así como se constituye el observador, como un vaso que no tiene fondo aparente, o quizás como un vaso cuya capacidad no alcanza a comprenderse en su totalidad, integrando todos los aprendizajes que ha experimentado en lo vivido.

Si un observador/profesional observa el fenómeno de la delincuencia, por ejemplo, los contenidos del observador mismo pueden ser muy diversos ¿Qué condiciones, características, conocimientos o competencias debiera poseer el observador? y ¿Qué/ Quién lo habilita para observar? En este caso existe un mandato institucional que valida a los profesionales a ejercer como observadores, considerando ciertas competencias, pero lo básico es que posea la certificación de formación académica en profesiones del área de ciencias sociales y no de otros ámbitos, como las ciencias exactas o naturales. Independiente de quien realice la observación, ésta se caracterizará por la subjetividad del observador. Ahora bien, resulta importante que independiente del trabajo profesional, el fenómeno de la delincuencia está siendo contenido por la estructura social en su conjunto, vale decir, por los profesionales, más los jueces, fiscales, defensores, policías e incluso la ciudadanía. Haciendo una analogía entre la observación de la delincuencia y la observación del mar, hay similitudes en el sentido de que la profundidad de la delincuencia y del mar no son perceptibles a una descripción superficial del fenómeno. Por ello, a medida que el observador se interna en esta profundidad, la capacidad predictiva puede ir disminuyendo ante el aumento de la complejidad. El profesional 
puede efectuar una descripción cercana a la fotografía de la marea, en base a la cual se pueden generar opiniones, acciones y caminos de la intervención. Reconocer que la observación no se acerca a la objetividad, motivará al profesional a situarse en un plano reflexivo de lo observado y de la forma de observar, lo cual no implica un relativismo, sólo el reconocimiento de los propios significados que contiene la subjetividad del observador.

El observador posee una posición determinada en el proceso de sanción a adolescentes infractores de ley, otorgada por la institución en el marco de la estructura social, la cual, junto al contexto de complejidad del fenómeno, constituye una parte de la subjetividad del profesional. Volviendo al ejemplo del mar, si lo observamos desde la costa en una posición segura, nos brinda mayor panorámica del fenómeno, pero también nos proporciona una posición fuera de éste. Por otro lado, la observación podría darse dentro del mar o del fenómeno social de la delincuencia, incorporando así al observador dentro del proceso, entendiendo que la interpretación de la complejidad de la delincuencia puede proporcionar nuevos datos de tipo etnográficos. Siguiendo con el ejemplo, el ejercicio de observar inmerso en el mar o en el fenómeno social de la delincuencia puede presentar dificultades, pero cada experiencia se constituye en algo único que aporta al profesional nuevos conocimientos y significados. Por ello, si el observador se reconoce como parte de la dinámica social, está asumiendo su subjetividad y la capacidad de permeabilidad en el proceso de intervención y vinculación.

\section{REFERENCIAS}

Aguirre, J., \& Jaramillo, L. (2010). La Ciencia entre el Objetivismo y el Construccionismo. Cinta Moebio, 38, 72-90.

Becker, H. (2012). Outsiders, hacia una sociología de la desviación. Buenos Aires: Siglo Veintiuno Editores.

Foucault, M. (2002). Vigilar y Castigar. Buenos Aires: Siglo Veintiuno.

García, J. (2008.) La conciencia del otro: Agresores y víctimas desde una perspectiva constructivista. Apuntes de Psicología, 26(2), 361-378.

Ley 20.084 que establece un Sistema de Responsabilidad de los Adolescentes por Infracciones a la Ley Penal (2005). Recuperado en http://www.leychile.cl/Navegar?idNorma=244803

Maturana, H. (1995). La ciencia y la vida cotidiana: La ontología de las explicaciones científicas. En P. Watzlawick \& P. Krieg (Comps.), El ojo del observador. Contribuciones al constructivismo (pp. 157-194). Barcelona: Gedisa.

Morin, E. (2011). Introducción al pensamiento complejo. Barcelona: Gedisa.

Morin, E. (2004). La epistemología de la complejidad. Gazeta de Antropología, 20, artículo 02. Recuperado de http://hdl.handle.net/10481/7253

Schaefer, H. (2014). Psicoterapias postestructuralistas y factores de cambio: Posibilidades 
para una práctica efectiva. Revista chilena de neuro-psiquiatría, 52(3), 177-184.

Szmulewicz, T. (2013). La persona del terapeuta: Eje fundamental de todo proceso terapéutico.

Revista chilena de neuro-psiquiatría, 51(1), 61-69.

Torrente, D. (2001). Desviación y delito. Madrid: Alianza Editorial.

White, M. (1993). Medios Narrativos para fines terapéuticos. Barcelona: Paidos Ibérica. 